\title{
AN ELEMENTARY PROOF OF THE BIEBERBACH CONJECTURE FOR THE SIXTH COEFFICIENT
}

\author{
By Mitsuru Ozawa
}

In our previous paper [2] we proved the Bieberbach conjecture for the sixth coefficient. Our proof did not lie on the elementary level, since we made use of Jenkins' inequality there. In this note we shall give an elementary proof of the Bieberbach conjecture for the sixth coefficient.

In the sequel we shall make use of the same notations as in [2]. From the methodological point of view there is nothing new in this note. Indeed we shall use the same method as in [2].

In [2] the main part was proved in the elementary method. Indeed we did not use Jenkins' inequality in Sections 2, 3, 4 and 5. Hence to give an elementary proof is rather trivial to the present author.

Section 6 was devoted to the case $1 \leqq p \leqq 1.6, k^{2} \leqq 1 / 10$. Then we have by the area theorem

$$
3\left(y^{2}+y^{\prime 2}\right) \leqq 4-p^{2} \leqq 3,
$$

which is better than Jenkins' inequality

$$
\sqrt{y^{2}+y^{\prime 2}} \leqq 1+2 e^{-6}<1.005 \text {. }
$$

This remark enables us to bring our proof into the elemetary level by slight modifications. And we can borrow the same estimations as they were in [2].

Section 7 was concerned with the case $1 \leqq p, 1 / 10 \leqq k^{2} \leqq 5-2 \sqrt{5}$. Again the area theorem gives a better inequality than Jenkins' in this case. Hence we can bring our proof into the elementary level by slight modifications and can borrow the same estimations as in [2].

Section 8 was concerned with the case $0 \leqq p \leqq 1, k^{2} \leqq 5-2 \sqrt{5}$. In this case the area theorem is not better than Jenkins'. Hence we need a new consideration. In this case we have

$$
3\left(y^{2}+y^{\prime 2}\right) \leqq 4-p^{2} \leqq 4
$$

in general. This implies that

$$
|y| \leqq 1.1548, \quad\left|y^{\prime}\right| \leqq 1.1548 .
$$

There were four subcases in Section 8.

Received July 23, 1968. 
Case 1). $y \geqq 0, k^{2} \leqq 1 / 29$. By starting from (A) with $B=0$, we have

$$
\begin{aligned}
\Re \alpha_{6} & \leqq \\
P(p)= & 6-\frac{2}{5}-\frac{2}{3} p^{2}-\frac{11}{120} p^{5}-\frac{25}{64} p^{4}(2-p)-\frac{p^{3}}{8} y \\
X= & \left(\frac{3}{2} p^{3}-\frac{7}{8} p x^{\prime 2}\right) x^{\prime 2}+\left(\frac{29}{8} p^{2}-\frac{11}{8} x^{\prime 2}\right) x^{\prime} y^{\prime}+\frac{7}{4} p y^{\prime 2}+\frac{7}{2} p x^{\prime} \eta^{\prime} \\
& +3 y^{\prime} \eta^{\prime}+2 x^{\prime} \xi^{\prime} .
\end{aligned}
$$

Now we have

$$
\begin{aligned}
P(p) & \geqq 6-\frac{2}{5}-\frac{2}{3} p^{2}-\frac{11}{120} p^{5}-\frac{25}{64} p^{4}(2-p)-\frac{1.1548}{8} p^{3} \\
& =6-\frac{1}{960} \phi(p), \\
\psi(p) & =384+640 p^{2}+138.576 p^{3}+750 p^{4}-287 p^{5} .
\end{aligned}
$$

It is easy to prove that $\phi(p) \leqq \psi(1)=1625.576$ for $0 \leqq p \leqq 1$. Therefore

$$
\begin{aligned}
P(p) & \geqq 6-\frac{1625.576}{960}=4.3+\frac{6.424}{960} \\
& >1.075\left(p^{2}+x^{\prime 2}+3 y^{2}+3 y^{\prime 2}+5 \eta^{2}+5 \eta^{\prime 2}+7 \xi^{\prime 2}\right) .
\end{aligned}
$$

Hence we have

$$
\begin{aligned}
\Re a_{6}< & 6-1.075 p^{2}-X^{*}+\frac{3}{4 \alpha} x^{\prime 2} y^{\prime 2}+\frac{3 \alpha}{4} y^{2}+\frac{5}{8 \beta} x^{\prime 4}+\frac{5 \beta}{8} \eta^{2} \\
& +\frac{7}{4} p y^{2}+3 y \eta-3.225 y^{2}-5.375 \eta^{2}, \\
X^{*}= & X+1.075\left(x^{\prime 2}+3 y^{\prime 2}+5 \eta^{\prime 2}+7 \xi^{\prime 2}\right), \quad \alpha>0, \beta>0 .
\end{aligned}
$$

Here we put $\alpha=1.3, \beta=1.4$. Then the quadratic form of $y, \eta$

$$
-\frac{1}{4}\left\{(9-7 p) y^{2}-12 y \eta+18 \eta^{2}\right\} \leqq 0
$$

for $0 \leqq p \leqq 1$. Hence

$$
\Re a_{6}<6-Q, \quad Q=X^{*}-\frac{3}{5.2} x^{\prime 2} y^{\prime 2}-\frac{5}{11.2} x^{\prime 4} .
$$

We make the associated symmetric matrix of $Q$ 


$$
\left(\begin{array}{llll}
17.2+24 p^{3}-14 p x^{\prime 2}-(50 / 7) x^{\prime 2} & 29 p^{2}-11 x^{\prime 2} & 28 p & 16 \\
29 p^{2}-11 x^{\prime 2} & 51.6+28 p-(12 / 1.3) x^{\prime 2} & 24 & 0 \\
28 p & 24 & 86 & 0 \\
16 & 0 & 0 & 120.4
\end{array}\right)
$$

It is easy to prove the positive definiteness of this matrix for $0 \leqq p \leqq 1, k^{2} \leqq 5-2 \sqrt{5}$ $<0.528$. Hence we have the desired result.

Case 2). $y \geqq 0, k^{2} \geqq 1 / 29$. In this case we have

$$
p^{3} y-29 p x^{\prime 2} y=29 p^{3}\left(\frac{1}{29}-k^{2}\right) y \leqq 0 .
$$

Hence we may omit them in this case. And we can apply the above estimation in Case 1).

Case 3). $y \leqq 0, k^{2} \leqq 1 / 29$. The same holds as in Case 2). Hence we can apply the above estimation in Case 1 ).

Case 4$)$. $y \leqq 0,1 / 29 \leqq k^{2}$. Firstly we assume that $0.5 \leqq p \leqq 1$. We start from (A) with $B=3 / 2$. Since

$$
-p y\left(28.4 x^{2}-15 p x\right)=-p^{2} y\left(28.4 k^{2} p-15 x\right) \leqq 0
$$

for $0 \leqq p \leqq 1, k^{2} \leqq 5-2 \sqrt{5}<0.528$, we have

$$
\begin{aligned}
& \Re a_{6} \leqq 6-P(p)-X-\frac{3}{2} x^{\prime} y^{\prime} y+\frac{9-7 p}{2} y^{2}-\frac{5}{4} x^{\prime 2} \eta+\frac{1}{8} p^{3} y-\frac{0.6}{8} p x^{\prime 2} y, \\
& P(p)=6-\frac{2}{5}-\frac{2}{3} p^{2}-\frac{11}{120} p^{5}-\frac{25}{64} p^{4}(2-p)
\end{aligned}
$$

with the same $X$ as in the above case. Further

$$
p^{3} y-0.6 p x^{\prime 2} y \leqq-y p^{3}(0.6 \cdot 0.528-1)<0
$$

for $k^{2} \leqq 5-2 \sqrt{5}<0.528$. Since $P(p) \geqq P(1)=4.451041 \dot{6}$ for $0.5 \leqq p \leqq 1$ and

$$
\begin{aligned}
\left|\frac{3}{2} x^{\prime} y^{\prime} y\right| & \leqq \frac{3}{4}\left|x^{\prime}\right|\left(y^{2}+y^{\prime 2}\right) \leqq \frac{4-p^{2}}{4}(0.528)^{1 / 2} \\
& \leqq \frac{1}{4}(0.528)^{1 / 2} 3.75 \leqq 0.6186
\end{aligned}
$$

we have

$$
\begin{aligned}
\Re a_{6} & <6-3.7694-X+\frac{5}{8 \beta} x^{\prime 4}+\frac{9-7 p}{2} y^{2}+\frac{5 \beta}{8} \eta^{2} \\
& <6-\frac{3.76}{4} p^{2}-Q(\beta)+\frac{1}{2}(9-7 p-5.64) y^{2}-\frac{1}{8}(37.6-5 \beta) \eta^{2}, \\
Q(\beta) & =X+\frac{3.76}{4}\left(x^{\prime 2}+3 y^{\prime 2}+5 \eta^{\prime 2}+7 \xi^{\prime 2}\right)-\frac{5}{8 \beta} x^{\prime 4}, \quad \beta>0 .
\end{aligned}
$$


The coefficient of $y^{2}$ is negative for $0.5 \leqq p \leqq 1$ and that of $\eta^{2}$ is negative by putting $\beta=7.5$. Hence we have

$$
\Re a_{6}<6-Q, \quad Q=Q(7.5) .
$$

It is easy to prove the positive definiteness of $Q$ for $k^{2} \leqq 5-2 \sqrt{5}, 0 \leqq p \leqq 1$. Hence we have the desired result.

Next we assume $0 \leqq p \leqq 0.5, k^{2} \leqq 5-2 \sqrt{5}$. We start from (A) with $B=0$. In this case

$$
-\frac{29}{8} p x^{\prime 2} y+\frac{p^{3}}{8} y \leqq \frac{1.1548}{8} p^{3}\left(29 k^{2}-1\right) \leqq \frac{1.1548}{64}(15.312-1)<0.25875
$$

and

$$
\left|-\frac{3}{2} x^{\prime} y^{\prime} y\right| \leqq \frac{3}{4}\left|x^{\prime}\right|\left(y^{2}+y^{\prime 2}\right) \leqq \frac{4-p^{2}}{4}|k| p<0.3635
$$

Hence we have

$$
\Re a_{6} \leqq 6-P(p)-X+\frac{7}{4} p y^{2}+3 y \eta-\frac{5}{4} x^{\prime 2} \eta+0.62225 .
$$

Since $P(p) \geqq P(0.5)>5.393836$ for $0 \leqq p \leqq 0.5$, we have, with $\alpha>0$,

$$
\begin{aligned}
\Re a_{6} & <6-4.77-X+\frac{5}{8 \alpha} x^{\prime 4}+\frac{5 \alpha}{8} \eta^{2}+\frac{7}{4} p y^{2}+3 y \eta \\
& \leqq 6-Q(\alpha)-Y(\alpha), \\
Q(\alpha) & =X-\frac{5}{8 \alpha} x^{\prime 4}+\frac{4.77}{4}\left(x^{\prime 2}+3 y^{\prime 2}+5 \eta^{\prime 2}+7 \xi^{\prime 2}\right), \\
4 Y(\alpha) & =(14.31-7 p) y^{2}-12 y \eta+(23.85-2.5 \alpha) \eta^{2} .
\end{aligned}
$$

Since $Y(8) \geqq 0$ for $\alpha=8$, we have

$$
\Re \alpha_{6}<6-Q, \quad Q=Q(8) .
$$

Now to prove the positive definiteness of $Q$ for $0 \leqq p \leqq 1, k^{2} \leqq 5-2 \sqrt{5}$ is very easy. Hence we have the desired result.

\section{REFERENCES}

[1] Jenkins, J. A., On certain coefficients of univalent functions, Analytic functions. Princeton Univ. Press (1960), 159-194.

[2] Ozawa, M.. On the Bieberbach conjecture for the sixth coefficient. Köda1 Math. Sem. Rep. 21 (1969), 97-128.

Department of Mathematics,

Tokyo Institute of Technology. 